\title{
Major clinical research advances in gynecologic cancer 2009
}

\author{
Kidong Kim, Sang-Young Ryu \\ Department of Obstetrics and Gynecology, Korea Cancer Center Hospital, Korea Institute of Radiological and Medical Sciences, Seoul, Korea
}

\begin{abstract}
In this review, we summarized nine major clinical advances in gynecology which occurred in 2009. For cervical cancer, the role of human papillomavirus (HPV) test as a screening test, the efficacy of HPV vaccine for middle-aged women, randomized controlled trial (RCT) regarding concurrent chemoradiation using gemcitabine plus cisplatin, and the efficacy of pazopanib for metastatic or recurrent disease were chosen. For endometrial cancer, the necessity of systematic pelvic lymphadenectomy in early endometrial cancer was reviewed. For ovarian cancer, the timing of treatment initiation for recurrent ovarian cancer, dose-dense chemotherapy as postoperative adjuvant treatment, the best chemotherapy regimen for platinum-sensitive recurrent ovarian cancer, and the efficacy of target agents were selected. In addition, the results of RCT testing the benefit of ginger in preventing post-chemotherapy nausea were examined.
\end{abstract}

Key Words: Gynecology, Urogenital neoplasms, Biomedical research

\section{INTRODUCTION}

In 2009, there were several notable advances in gynecologic oncology field. Generally, the studies investigating the efficacy of target agents are increasing year by year. However, there were also remarkable advances in optimization of treatment using conventional anti-neoplastic therapies such as chemotherapy drugs or radiation.

Topics included in this review were selected by reviewing the literatures and abstracts which were published or presented in 2009. Specifically, we examined abstracts which were presented at oral or plenary sessions of the American Society of Clinical Oncology (ASCO) annual meeting, the Society of Gynecology Oncology (SGO) annual meeting, the International Federation of Gynecology and Obstetrics (FIGO) biennial meeting, and the European Society of Gynecological Oncology (ESGO) biennial meeting. In addition, we searched the news at educational sites such as Medscape (www.medscape.com) or Clinical Care Options (www.clinicaloptions.com), and reviewed the titles of article which were published at major journals in 2009.

The objective of this review was to summarize the major clinical research advances in gynecologic cancer and to help

Received December 9, 2009, Accepted December 9, 2009

Correspondence to Sang-Young Ryu

Department of Obstetrics and Gynecology, Korea Cancer Center Hospital, Korea Institute of Radiological and Medical Sciences, Gongneung-dong, Nowon-gu, Seoul 139-706, Korea

Tel: 82-2-970-1227, Fax: 82-2-970-1227

E-mail: ryu@kcch.re.kr reader update their knowledge.

\section{MAJOR CLINICAL RESEARCH ADVANCES}

1. Human papillomavirus testing as a screening test for cervical cancer

Cytological examination of cervical cells (Pap test and liquidbased cytology) has been the standard screening test for cervical cancer. According to several observational studies, the cytological test has reduced the incidence of cervical cancer in many countries. ${ }^{1,2}$ However, the cytological test has limitations such as low sensitivity and poor reproducibility.,

The major cause of cervical cancer is the infection with human papillomavirus (HPV) and the sustained infection with highrisk HPV precedes the developments of cervical intraepithelial neoplasia (CIN) and cervical cancer. Therefore, the test for HPV infection could detect the women who are at risk to develop a CIN and cervical cancer.

Several randomized controlled trials (RCTs) have compared the efficacy of HPV test with that of cytological test in screening for cervical cancer. These trials demonstrated that the HPV test has higher sensitivity than the cytological test but lower specificity. The lower specificity of HPV test caused more unnecessary testings and visits than cytological test. The major cause of lower specificity of HPV test was the higher prevalence and regression rate of HPV infection in young women., ${ }^{3,5,6}$ In 2009, there were two notable studies advocating the value of HPV test as a primary screening test for cervical cancer. In a study, researchers hypothesized that the low specificity of HPV test could be overcome by alternative combination of HPV and cytological tests. ${ }^{7}$ Using the data of Swedescreen trial, ${ }^{6,8}$ 
the researchers examined the efficacy of 11 different strategies alternatively combining HPV and cytological tests. In result, the most effective strategy was HPV test followed by cytological triage and repeat HPV test for HPV positive women with normal cytology. Compared with cytology alone, the 'winner' strategy increased the sensitivity for CIN 3 or worse by $30 \%$, maintained a high positive predictive value, and resulted in an only $12 \%$ increase in the number of screening tests. $^{7}$

In the other study, researchers examined the effect of a single round of screening by HPV test, cytological test, visual inspection of cervix with acetic acid, or standard care on the incidence of cervical cancer and the associated rates of death in a district of India. The trial included 131,746 healthy women between ages of 30 and 59 years. Compared with control (standard care), the HPV test reduced the risk of advanced cancer by $53 \%$ and the associated rates of death by $48 \%$. However, the cytological test and visual inspection did not reduce the numbers of advanced cancer and deaths. ${ }^{9}$ Considering that most cervical cancers occur in low-resource area such as India, we think that the adoption of HPV test as a primary screening test for cervical cancer has the potential to reduce the global burden of cervical cancer.

While the most effective and feasible strategy for cervical cancer screening should be tested in individual age groups and countries, we believe that the shift from cytological test to HPV test would occur in near future.

\section{HPV vaccine for middle-aged women}

HPV vaccine is a prophylactic vaccine targeting HPV L1 viruslike particle and was shown to be highly effective in prevention of cervical, vulvar, or vaginal intraepithelial neoplasia related to HPV $6,11,16,18 .^{10,11}$ Because the RCTs verifying the efficacy of HPV vaccines only included women younger than 27 , there were no direct evidences to support the vaccination to women older than 26. However, from our experience, most of women visiting the clinic for HPV vaccination is older than 26 . Therefore, it was a dilemma whether the physician should recommend the HPV vaccination for women older than 26.

In 2009, the results of RCT demonstrating the efficacy of HPV vaccines in women aged $24-45$ years were published. A total of 3,819 women with no history of genital warts or cervical disease were randomized to quadrivalent HPV vaccine or placebo. In per-protocol analysis, the quadrivalent HPV vaccine was $90.5 \%$ effective in preventing infection and disease related to HPV 6, 11, 16, 18 and was $83.1 \%$ effective in preventing infection and disease related to HPV 16, 18. However, the efficacy in intention-to-treat population was quite low due to the infection and disease being present at baseline. Specifically, the efficacy against infection and disease related to HPV 6, 11, 16,18 was $30.9 \%$ and that against HPV 16,18 was $22.6 \%{ }^{12}$ Because the mean follow-up time of this RCT is only 2.2 years, we do not know whether the HPV vaccine could provide the long-term protection for women aged 24-45. The RCT is on- going to determine the duration of protection against cervical cancer.

The result of this RCT is the first direct evidence for efficacy of HPV vaccine in middle-aged women. While the issues such as the duration of protection and cost-effectiveness are unsolved, we believe that we should recommend HPV vaccination to women aged 24-45.

\section{Concurrent chemoradiation using gemcitabine plus cisplatin for cervical cancer}

The standard treatment for locally advanced cervical cancer is the concurrent chemoradiation (CRT), and several chemotherapy regimens have been evaluated to find the most effective regimen. ${ }^{13}$ According to Gynecologic Oncology Group (GOG) protocol 120, weekly intravenous cisplatin $40 \mathrm{mg} / \mathrm{m}^{2}$ for 6 weeks was more convenient, equally effective and less toxic than 5-fluorouracil, cisplatin plus hydroxyurea regimen. ${ }^{14}$ Thereafter, the weekly cisplatin plus radiation was established as the new standard for the treatment of locally advanced cervical cancer, and all subsequent GOG RCTs for locally advanced cervical cancer have used this regimen as the standard arm. ${ }^{15}$ In the 2009 ASCO annual meeting, the results of RCT testing the efficacy of CRT using alternative chemotherapy regimen for locally advanced cervical cancer were presented. Chemotherapy- and radiation-naive 515 patients with bulky stage 2B-4A cervical cancer were randomized to two groups. The patients in experimental arm received six cycles of weekly cisplatin $40 \mathrm{mg} / \mathrm{m}^{2}$ plus gemcitabine $125 \mathrm{mg} / \mathrm{m}^{2}$ with concurrent external radiation (50.4 Gy in 28 fraction) followed by brachytherapy (30-35 Gy) and two cycles of adjuvant triweekly gemcitabine plus cisplatin (gemcitabine $1,000 \mathrm{mg} / \mathrm{m}^{2}$ on days 1 and 8 ; cisplatin $50 \mathrm{mg} / \mathrm{m}^{2}$ on day 1 ). The patients in control arm received six cycles of weekly cisplatin $40 \mathrm{mg} / \mathrm{m}^{2}$ with concurrent external radiation followed by brachytherapy. The dose and schedule of radiation in control arm were same as that of experimental arm. The compliance to experimental regimen was acceptable. Specifically, over $90 \%$ of patients in experimental arm completed the external radiation plus brachytherapy. In addition, over $75 \%$ of patients in experimental arm completed two cycles of adjuvant triweekly gemcitabine and cisplatin. The progression-free survival (PFS) at three years was $74 \%$ in experimental arm and $65 \%$ in control $\operatorname{arm}(p=0.029)$. The overall survival (OS; $p=0.022)$ and time to progressive disease $(\mathrm{p}<0.001)$ were also significantly improved in the experimental arm. However, grade $3 / 4$ toxicities were more frequently observed in experimental arm (86.5\%) than in control arm (46.3\%). In addition, two patients in experimental arm died due to causes probably related to treatment. $^{16}$

While the new regimen reported the longer survival than the current standard regimen, it is unclear which components of new regimen contributed to survival benefit. Specifically, the survival benefit of new regimen could be the effect of weekly gemcitabine plus cisplatin or that of two cycles of adjuvant 
chemotherapy. Nevertheless, based on the survival benefit and acceptable toxicities, we believe that the gemcitabine plus cisplatin regimen should be one of the standard treatment regimens for locally advanced cervical cancer.

\section{Pazopanib for metastatic or recurrent cervical cancer}

The current standard treatment for metastatic or recurrent cervical cancer is platinum-based chemotherapy with or without radiation. ${ }^{17}$ However, the prognosis of patients with metastatic or recurrent cervical cancer is known to be poor. Specifically, in the trial comparing four platinum-based doublet chemotherapies for patients with metastatic or recurrent cervical cancer, the median OS of the reference arm was only 13 months. ${ }^{18}$ Therefore, numerous novel agents have been tested to improve the prognosis of patients with metastatic or recurrent cervical cancer.

In the 2009 ASCO annual meeting, the results of randomized phase 2 trial evaluating the efficacy of two novel tyrosine kinase inhibitors (pazopanib and lapatinib) in metastatic or recurrent cervical cancer were presented. Pazopanib targets vascular endothelial growth factor receptor, platelet-derived growth factor receptor, and c-Kit; lapatinib targets epidermal growth factor receptor. A total of 235 patients with stage 4B, persistent or recurrent cervical cancer were randomized to pazopanib $800 \mathrm{mg}$ daily, lapatinib 1,500 mg daily, or pazopanib $400 \mathrm{mg}$ plus lapatinib $1000 \mathrm{mg}$ daily. In result, pazopanib monotherapy improved PFS (hazard ratio [HR], 0.66; 90\% confidence interval [CI], 0.48 to 0.91 ) and OS (HR, 0.67; 90\% CI, 0.46 to 0.99 ) compared to lapatinib. The most common grade 3 or more adverse event was diarrhea ( $11 \%$ for pazopanib and $13 \%$ for lapatinib). ${ }^{19}$

Because this was the phase 2 trial and did not include the standard treatment arm (chemotherapy), it is hard to know pazopanib is more effective than the current standard treatment. However, the excellent anti-neoplastic efficacy, oral administration, and low incidence of serious toxicities made pazopanib an attractive candidate for further evaluation.

\section{Necessity of systematic pelvic lymphadenectomy in endometrial cancer}

Whether we should perform the systematic pelvic lymphadenectomy (SPL) for patients with clinically early endometrial cancer has been a controversial issue. Until a recent date, there were no definitive results from well-designed RCTs comparing the outcome of extrafascial hysterectomy plus bilateral adnexectomy $(\mathrm{H}+\mathrm{BSO})$ with that of $\mathrm{H}+\mathrm{BSO}+\mathrm{SPL}$. Recently, the results from two RCTs directly addressing this issue were published.

Medical Research Council A Study in the Treatment of Endometrial Cancer (MRC ASTEC) trial included 1,408 patients with histologically proven endometrial cancer which were thought preoperatively to be confined to the corpus. The enrolled patients were randomized to standard surgery $(\mathrm{H}+$ $\mathrm{BSO}$, peritoneal washings, and palpation of para-aortic lymph nodes) or standard surgery plus SPL. Patients who had a computed tomography (CT) or magnetic resonance imaging (MRI) scan suggesting node enlargement were not excluded from the trial and nodes that were suspicious could be sampled in the standard surgery arm. The number of patients who received adjuvant radiation was similar between the two arms. The OS of standard surgery arm was similar with that of SPL arm (HR, 1.16; $95 \%$ CI, 0.87 to 1.54). Furthermore, the recurrence-free survival (RFS) of standard surgery arm was longer than that of SPL arm (HR, 1.35; 95\% CI, 1.06 to 1.73). Therefore, researchers concluded that there is no evidence of benefit in terms of OS or RFS for SPL in patients with early endometrial cancer. ${ }^{20}$

The other RCT regarding the role of SPL in early endometrial cancer was conducted by the Italian group. From 1996 to 2006, 514 patients with preoperative stage 1 endometrial cancer were randomly assigned to SPL or no lymphadenectomy arms. Patients with stage $1 \mathrm{~A} / 1 \mathrm{~B}$ and grade 1 cancer based on intraoperative frozen section diagnosis were excluded. Bulky $(>1 \mathrm{~cm})$ lymph node detected by intraoperative palpation of lymph node sites were permitted to remove in no lymphadenectomy arm. The OS and disease-free survival (DFS) of SPL arm were similar with those of no lymphadenectomy arm (for OS, HR, 1.20 and $95 \%$ CI, 0.70 to 2.07; for DFS, HR, 1.10 and $95 \% \mathrm{CI}, 0.70$ to 1.71$)^{21}$

Although these two RCTs have limitations that the lymphadenectomy specified in the protocol did not include para-aortic lymph nodes, it seemed clear that the SPL had no therapeutic value in early endometrial cancer. Considering that SPL did not improve the survival but increased the morbidities related to surgery, we believe that SPL should not be recommended as routine procedure in early endometrial cancer.

\section{Early versus delayed treatment of recurrent ovarian cancer}

The serial measurement of serum CA 125 is a sensitive method to detect a recurrence in patients with ovarian cancer. According to a study which including 255 patients with ovarian cancer, a doubling of CA 125 levels from the upper limit of normal had a sensitivity of $86 \%$, specificity of $91 \%$, positive predictive value $95 \%$, and negative predictive value $78 \%$ for progression. ${ }^{22}$ Based on these findings, the gynecologic cancer intergroup (GCIG) proposed that the elevated CA 125 levels should be regarded as a progression of disease. ${ }^{23}$

In addition, the rising CA 125 levels are known to precede the clinical detection of recurrence with a median lead time of 3-5 months. ${ }^{22,24,25}$ Although there is no demonstration that an early detection of recurrence is beneficial, many clinical guidelines recommended the serial measurements of CA 125 every 2-4 months in patients with ovarian cancer. Furthermore, some physicians advocated initiating the chemotherapy for asymptomatic patients with elevated CA 125 levels. ${ }^{26}$

In the 2009 ASCO annual meeting, the results of RCT testing the benefit of early treatment based on elevated CA 125 levels compared to delayed treatment after clinical or symptomatic 
recurrence were presented. Patients with ovarian cancer in clinical complete remission after the first-line platinum-based chemotherapy were included in the RCT. CA 125 level was measured every three months but patients and investigators were blinded to the results. When the CA 125 levels exceeded twice the upper limit of normal, patients were randomized to either immediate treatment or to delayed treatment when clinical or symptomatic recurrence appeared. The chemotherapy for recurrence started a median of five months earlier in the immediate treatment arm. However, with a median follow-up of 49 months from randomization, the OS of immediate treatment arm was similar with that of delayed treatment arm (HR, 1.01; 95\% CI, 0.82 to 1.25). Furthermore, the patients in immediate treatment arm reported worse quality of life than those in delayed treatment arm. The researchers concluded that there is no value in the routine measurement of CA 125 in the follow-up of ovarian cancer patients. ${ }^{27}$

While the value of CA 125 measurement was negated by the RCT, we believe that the complete abandonment of CA 125 measurement could be a hasty conclusion because the RCT has some limitations. For example, the rates of optimal versus suboptimal secondary cytoreduction were not addressed and the patients in both arms did not receive the same treatment after recurrence. Therefore, the impact of this RCT on clinical practice and on guidelines for post-therapy surveillance remain to be determined.

\section{Dose-dense chemotherapy as postoperative adjuvant treatment}

The standard postoperative treatment for advanced ovarian cancer is triweekly intravenous (IV) paclitaxel plus carboplatin. Several modifications of postoperative adjuvant therapy have been attempted to improve survival but yielded unsatisfactory results. ${ }^{28}$ In the ASCO 2008 annual meeting, the preliminary results of RCT showing the superiority of dose dense weekly administration of paclitaxel plus carboplatin over current standard triweekly regimen was presented ${ }^{29}$ and we cited the results in our previous review. ${ }^{30}$ In 2009 , the mature data of the RCT was published. ${ }^{28}$

In Japan, a total of 631 patients with stage 2 to 4 epithelial ovarian cancer, fallopian tube cancer, or primary peritoneal cancer were randomly assigned to receive six cycles of either weekly paclitaxel $\left(80 \mathrm{mg} / \mathrm{m}^{2}\right.$; 1 hour IV infusion; on days $1,8,15$ of 21-day cycle) plus triweekly carboplatin (area under curve [AUC] 6 on day 1) or triweekly paclitaxel $\left(180 \mathrm{mg} / \mathrm{m}^{2} ; 3\right.$ hours infusion; on day 1) plus carboplatin (AUC 6 on day 1). In results, median PFS was longer in the weekly arm (28 months) than in the triweekly arm (17 months) and OS at three years was higher in the weekly arm (72\%) than in the triweekly arm $(65 \%$; HR, 0.75 ; $95 \%$ CI, 0.57 to 0.98 ). The most common toxicity was neutropenia in both arms and the frequency of grade $3 / 4$ anemia was higher in the weekly arm. However, the frequencies of other toxicities were similar between arms. ${ }^{28}$

Three years ago, GOG has finished a RCT (GOG 170) regarding intraperitoneal (IP) chemotherapy in ovarian cancer and reported the survival advantage of IP arm. The IP regimen was paclitaxel $135 \mathrm{mg} / \mathrm{m}^{2}$ IV over 24 -h on day 1 plus cisplatin $100 \mathrm{mg} / \mathrm{m}^{2} \mathrm{IP}$ on day 2 followed by paclitaxel $60 \mathrm{mg} / \mathrm{m}^{2}$ IP on day 8 of 21 -day cycle. ${ }^{31}$ Therefore, both the IP arm of GOG 170 and weekly arm of Japanese RCT adopted weekly paclitaxel administration. Until the results of Japanese RCT were reported, we thought that the survival advantage of IP arm of GOG 170 was due to the difference in drug infusion method (IP versus IV). However, considering the remarkable survival advantage of weekly paclitaxel shown in Japanese RCT, we think that the survival advantage of IP arm in GOG 170 could be attributed to weekly administration of paclitaxel not to IP infusion of drugs.

Confirmatory trials are planned by member groups of GCIG to evaluate weekly paclitaxel plus triweekly carboplatin regimen with and without bevacizumab. The results of confirmatory trials would decide whether the weekly paclitaxel plus triweekly carboplatin regimen would be a new standard treatment for advanced ovarian cancer.

\section{Best chemotherapy regimen for platinum-sensitive recurrent ovarian cancer}

There have been four RCTs comparing the efficacy of combination chemotherapy with that of single agent chemotherapy in patients with platinum-sensitive recurrent ovarian cancer. Of four RCTs, two RCTs compared paclitaxel plus platinum with platinum alone and showed the results favoring paclitaxel plus platinum over platinum alone. ${ }^{32}$ Another RCT reported the survival benefit of pegylated liposomal doxorubicin plus carboplatin compared to carboplatin alone but the results were based on accrual of only $61 \%$ of a planned 900 women. ${ }^{33}$ The other RCT demonstrated the PFS benefit of gemcitabine plus carboplatin compared to carboplatin alone. ${ }^{34}$ However, there have been no RCTs comparing the combination chemotherapy regimens each other.

In the 2009 ASCO annual meeting, the preliminary results of RCT comparing pegylated liposomal doxorubicin plus carboplatin with paclitaxel plus carboplatin were presented. In the RCT, 976 patients with platinum-sensitive recurrent ovarian cancer were randomized to either pegylated liposomal doxorubicin $30 \mathrm{mg} / \mathrm{m}^{2}$ IV plus carboplatin AUC 5 IV every four weeks or paclitaxel $175 \mathrm{mg} / \mathrm{m}^{2}$ IV plus carboplatin AUC 5 IV every three weeks. The PFS of pegylated liposomal doxorubicin arm was longer than that of paclitaxel arm (HR, 0.82; $95 \% \mathrm{CI}, 0.72$ to 0.94 ). In addition, patients in pegylated liposomal doxorubicin arm experienced less alopecia and neuropathy but more thrombocytopenia and hand-foot syndrome. ${ }^{35}$

Considering the higher PFS and different toxicity profile, we believe that the pegylated liposomal doxorubicin plus carboplatin could be an alternative to paclitaxel plus carboplatin. For example, for patients who fear for alopecia or have a neuropathy, pegylated liposomal doxorubicin plus carboplatin regimen would be useful. 


\section{Target agents for ovarian cancer}

Ovarian cancer is the most lethal gynecologic cancer. Nearly $70-80 \%$ of patients with advanced ovarian cancer eventually experienced a recurrence and most of patients with recurrent ovarian cancer died of disease. ${ }^{36}$ Therefore, numerous novel drugs have been tested for improving the prognosis of patients with ovarian cancer.

Olaparib is an oral poly ADP ribose polymerase (PARP) inhibitor and was reported to kill breast cancer (BRCA)-mutated cancer cells. ${ }^{37}$ In the 2009 ASCO annual meeting, the results of phase 2 trial evaluating the efficacy of olaparib in patients with recurrent ovarian cancer and mutated BRCA genes were presented. For 57 patients, olaparib $400 \mathrm{mg}$ twice daily $(\mathrm{N}=33)$ or $100 \mathrm{mg}$ twice daily $(\mathrm{N}=24)$ were given continuously. Although the trial included heavily pretreated patients, the response rate was $33 \%$ at $400 \mathrm{mg}$ twice daily dose and toxicities were mild. ${ }^{38}$

Aflibercept, also known as VEGF-TRAP, is a recombinant fusion protein and potent inhibitor of VEGF. ${ }^{39}$ The results of RCT evaluating the efficacy of Aflibercept in reducing malignant ascites caused by recurrent ovarian cancer were presented at the 2009 ASCO annual meeting. Fifty-five Patients with advanced, chemotherapy-resistant ovarian cancer suffering from symptomatic malignant ascites requiring 1-4 paracentesis per month were randomized to Aflibercept or placebo. Patients could cross-over and receive open-label Aflibercept after 60 days. In result, Aflibercept showed significant efficacy in the control of malignant ascites. Specifically, Aflibercept increased the mean time to first repeat paracentesis and reduced the number of paracentesis. However, the OS was similar between Aflibercept and placebo arms. Furthermore, three intestinal perforations were observed in Aflibercept arm. ${ }^{40}$

Until now, no target agents have been accepted as a standard therapy for ovarian cancer. However, the number of trials testing the efficacy of target agents for ovarian cancer is rapidly increasing and the results of some trials are promising. We believe that, in near future, target agents will replace the conventional chemotherapy and enable the more individualized therapy.

\section{Ginger for chemotherapy-related nausea}

Nausea and vomiting is one of the most common toxicities from chemotherapy. Drugs such as cisplatin, carboplatin, and doxorubicin which are commonly used for gynecological cancer patients are known to be highly emetogenic. Therefore, control of nausea and vomiting is a major issue for gynecologic oncologists.

While the 5-HT3 receptor antagonist antiemetics are widely used, the post-chemotherapy nausea and vomiting continue to be experienced by patients who are receiving highly emetogenic therapies. For example, data from University of Rochester Cancer Center Community Clinical Oncology Program Research Base study showed that $77 \%$ of patients with highly emetogenic therapies experienced delayed nausea and $20-30 \%$ of patients suffered anticipatory nausea or vomiting. ${ }^{41,42}$ Therefore, it is necessary to develop new modalities to control postchemotherapy nausea and vomiting.

In the 2009 ASCO annual meeting, the results of RCT testing the efficacy of ginger in alleviating the post-chemotherapy nausea were presented. Cancer patients who experienced nausea following any chemotherapy and were scheduled to receive at least three additional cycles were registered. A total of 644 patients were randomized into four arms: placebo, 0.5 g ginger, $1.0 \mathrm{~g}$ ginger, or $1.5 \mathrm{~g}$ ginger. All patients received 5-HT3 receptor antagonist antiemetics on day 1 of all cycles and took ginger capsules for six days starting three days before the first day of cycles. Patients reported the severity of nausea on a 7-point semantic rating scale for days 1-4 of each cycle. Patients with several types of cancer were included in the RCT and the most common type of cancer was breast cancer. In result, all doses of ginger significantly reduced nausea and the largest reduction in nausea occurred with $0.5 \mathrm{~g}$ and $1.0 \mathrm{~g}$ of ginger. $^{43}$

Although the pharmacology of ginger has not been studied extensively in humans and the mechanism of action by which ginger reduce nausea and vomiting is not completely known, ${ }^{44}$ most Koreans are familiar to ginger because ginger has been widely used as a flavoring agent for food in Korea. Considering the food style in Korea, we think that studies using gingercontaining food or tea in prevention of post-chemotherapy nausea would be interesting.

\section{CONCLUSION}

Since 2007, every year, we have reviewed advances in gynecologic cancer research and summarized topics which have had the greatest impact on patient care. In 2009, we think, the most notable change was the increase of the trials testing the efficacy of target agents. Several agents such as olaparib showed promising efficacy against gynecologic cancer. We believe that more agents will be developed and be tested in following years. Furthermore, in near future, target agents could replace the conventional chemotherapy or radiation. Eventually, target agents will enable us to perform more individualized therapies.

\section{REFERENCES}

1. Bergstrom R, Sparen P, Adami HO. Trends in cancer of the cervix uteri in Sweden following cytological screening. $\mathrm{Br} \mathrm{J}$ Cancer 1999; 81: 159-66.

2. Gustafsson L, Ponten J, Zack M, Adami HO. International incidence rates of invasive cervical cancer after introduction of cytological screening. Cancer Causes Control 1997; 8: 755-63.

3. Mayrand MH, Duarte-Franco E, Rodrigues I, Walter SD, Hanley J, Ferenczy A, et al. Human papillomavirus DNA versus Papanicolaou screening tests for cervical cancer. N Engl J Med 2007; 357: 1579-88.

4. Nanda K, McCrory DC, Myers ER, Bastian LA, Hasselblad V, Hickey JD, et al. Accuracy of the Papanicolaou test in screening for and follow-up of cervical cytologic abnormalities: a systematic review. Ann Intern Med 2000; 132: 810-9. 
5. Bulkmans NW, Berkhof J, Rozendaal L, van Kemenade FJ, Boeke AJ, Bulk S, et al. Human papillomavirus DNA testing for the detection of cervical intraepithelial neoplasia grade 3 and cancer: 5-year follow-up of a randomised controlled implementation trial. Lancet 2007; 370: 1764-72.

6. Naucler P, Ryd W, Tornberg S, Strand A, Wadell G, Elfgren K, et al. Human papillomavirus and Papanicolaou tests to screen for cervical cancer. N Engl J Med 2007; 357: 1589-97.

7. Naucler P, Ryd W, Tornberg S, Strand A, Wadell G, Elfgren K, et al. Efficacy of HPV DNA testing with cytology triage and/or repeat HPV DNA testing in primary cervical cancer screening. J Natl Cancer Inst 2009; 101: 88-99.

8. Elfgren K, Rylander E, Radberg T, Strander B, Strand A, Paajanen $\mathrm{K}$, et al. Colposcopic and histopathologic evaluation of women participating in population-based screening for human papillomavirus deoxyribonucleic acid persistence. Am J Obstet Gynecol 2005; 193: 650-7.

9. Sankaranarayanan R, Nene BM, Shastri SS, Jayant K, Muwonge $\mathrm{R}$, Budukh AM, et al. HPV screening for cervical cancer in rural India. N Engl J Med 2009; 360: 1385-94.

10. FUTURE II Study Group. Quadrivalent vaccine against human papillomavirus to prevent high-grade cervical lesions. N Engl J Med 2007; 356: 1915-27.

11. Garland SM, Hernandez-Avila M, Wheeler CM, Perez G, Harper DM, Leodolter S, et al. Quadrivalent vaccine against human papillomavirus to prevent anogenital diseases. N Engl J Med 2007; 356: 1928-43.

12. Munoz N, Manalastas R Jr, Pitisuttithum P, Tresukosol D, Monsonego J, Ault K, et al. Safety, immunogenicity, and efficacy of quadrivalent human papillomavirus (types $6,11,16,18$ ) recombinant vaccine in women aged $24-45$ years: a randomised, double-blind trial. Lancet 2009; 373: 1949-57.

13. Koh WJ. Controversies in the radiotherapeutic management of cervical cancer. J Clin Oncol 2003; 21 (10 Suppl): 218S-23S.

14. Rose PG, Bundy BN, Watkins EB, Thigpen JT, Deppe G, Maiman MA, et al. Concurrent cisplatin-based radiotherapy and chemotherapy for locally advanced cervical cancer. N Engl J Med 1999; 340: 1144-53.

15. Monk BJ, Tian C, Rose PG, Lanciano R. Which clinical/pathologic factors matter in the era of chemoradiation as treatment for locally advanced cervical carcinoma? Analysis of two Gynecologic Oncology Group (GOG) trials. Gynecol Oncol 2007; 105: 427-33.

16. Duenas-Gonzalez A, Zarba JJ, Alcedo JC, Pattarunataporn P, Beslija S, Patel F, et al. A phase III study comparing concurrent gemcitabine (Gem) plus cisplatin (Cis) and radiation followed by adjuvant Gem plus Cis versus concurrent $\mathrm{Cis}$ and radiation in patients with stage IIB to IVA carcinoma of the cervix. 2009 ASCO Annual Meeting, Abstract CRA5507.

17. Monk BJ, Willmott LJ, Sumner DA. Anti-angiogenesis agents in metastatic or recurrent cervical cancer. Gynecol Oncol 2009. Epub 2009 Oct 25.

18. Monk BJ, Sill MW, McMeekin DS, Cohn DE, Ramondetta LM, Boardman $\mathrm{CH}$, et al. Phase III trial of four cisplatin-containing doublet combinations in stage IVB, recurrent, or persistent cervical carcinoma: a Gynecologic Oncology Group study. J Clin Oncol 2009; 27: 4649-55.

19. Monk B, Mas L, Zarba JJ, Oaknin A, Tarpin C, Termrungruanglert $\mathrm{W}$, et al. A randomized phase II study: Pazopanib (P) versus lapatinib $(\mathrm{L})$ versus combination of pazopanib/lapatinib $(\mathrm{L}+\mathrm{P})$ in advanced and recurrent cervical cancer (CC). 2009 ASCO Annual Meeting, Abstract 5520.

20. Kitchener H, Swart AM, Qian Q, Amos C, Parmar MK. Efficacy of systematic pelvic lymphadenectomy in endometrial cancer (MRC ASTEC trial): a randomised study. Lancet 2009; 373: 125-36.
21. Benedetti Panici P, Basile S, Maneschi F, Alberto Lissoni A, Signorelli M, Scambia G, et al. Systematic pelvic lymphadenectomy vs. no lymphadenectomy in early-stage endometrial carcinoma: randomized clinical trial. J Natl Cancer Inst 2008; 100: 1707-16.

22. Rustin GJ, Nelstrop AE, Tuxen MK, Lambert HE. Defining progression of ovarian carcinoma during follow-up according to CA 125: a North Thames Ovary Group Study. Ann Oncol 1996; 7: 361-4.

23. Vergote I, Rustin GJ, Eisenhauer EA, Kristensen GB, PujadeLauraine E, Parmar MK, et al. Re: new guidelines to evaluate the response to treatment in solid tumors [ovarian cancer]. Gynecologic Cancer Intergroup. J Natl Cancer Inst 2000; 92: 1534-5.

24. Gadducci A, Cosio S, Carpi A, Nicolini A, Genazzani AR. Serum tumor markers in the management of ovarian, endometrial and cervical cancer. Biomed Pharmacother 2004; 58: 24-38.

25. van der Burg ME, Lammes FB, Verweij J. The role of CA 125 and conventional examinations in diagnosing progressive carcinoma of the ovary. Surg Gynecol Obstet 1993; 176: 310-4.

26. Gadducci A, Cosio S. Surveillance of patients after initial treatment of ovarian cancer. Crit Rev Oncol Hematol 2009; 71: 43-52.

27. Rustin GJ, van der Burg ME. A randomized trial in ovarian cancer (OC) of early treatment of relapse based on CA125 level alone versus delayed treatment based on conventional clinical indicators (MRC OV05/EORTC 55955 trials). 2009 ASCO Annual Meeting, Abstract 1.

28. Katsumata N, Yasuda M, Takahashi F, Isonishi S, Jobo T, Aoki $\mathrm{D}$, et al. Dose-dense paclitaxel once a week in combination with carboplatin every 3 weeks for advanced ovarian cancer: a phase 3, open-label, randomised controlled trial. Lancet 2009; 374: 1331-8.

29. Isonishi S, Yasuda M, Takahashi F, Katsumata N, Kimura E, Aoki D, et al. Randomized phase III trial of conventional paclitaxel and carboplatin (c-TC) versus dose dense weekly paclitaxel and carboplatin (dd-TC) in women with advanced epithelial ovarian, fallopian tube, or primary peritoneal cancer: Japanese Gynecologic Oncology. 2008 ASCO Annual Meeting, Abstract 5506.

30. Kim K, Choi SC, Ryu SY, Kim JW, Kang SB. Major clinical research advances in gynecologic cancer 2008. J Gynecol Oncol 2008; 19: 209-17.

31. Armstrong DK, Bundy B, Wenzel L, Huang HQ, Baergen R, Lele $\mathrm{S}$, et al. Intraperitoneal cisplatin and paclitaxel in ovarian cancer. N Engl J Med 2006; 354: 34-43.

32. Parmar MK, Ledermann JA, Colombo N, du Bois A, Delaloye JF, Kristensen GB, et al. Paclitaxel plus platinum-based chemotherapy versus conventional platinum-based chemotherapy in women with relapsed ovarian cancer: the ICON4/AGO-OVAR2.2 trial. Lancet 2003; 361: 2099-106.

33. Alberts DS, Liu PY, Wilczynski SP, Clouser MC, Lopez AM, Michelin DP, et al. Randomized trial of pegylated liposomal doxorubicin (PLD) plus carboplatin versus carboplatin in platinum-sensitive (PS) patients with recurrent epithelial ovarian or peritoneal carcinoma after failure of initial platinum-based chemotherapy (Southwest Oncology Group Protocol S0200). Gynecol Oncol 2008; 108: 90-4.

34. Pfisterer J, Plante M, Vergote I, du Bois A, Hirte H, Lacave AJ, et al. Gemcitabine plus carboplatin compared with carboplatin in patients with platinum-sensitive recurrent ovarian cancer: an intergroup trial of the AGO-OVAR, the NCIC CTG, and the EORTC GCG. J Clin Oncol 2006; 24: 4699-707.

35. Pujade-Lauraine E, Mahner S, Kaern J, Gebski V, Heywood M, 
Vasey P, et al. A randomized, phase III study of carboplatin and pegylated liposomal doxorubicin versus carboplatin and paclitaxel in relapsed platinum-sensitive ovarian cancer (OC): CALYPSO study of the Gynecologic Cancer Intergroup (GCIG). 2009 ASCO Annual Meeting, Abstract LBA5509.

36. Young RC, Decker DG, Wharton JT, Piver MS, Sindelar WF, Edwards BK, et al. Staging laparotomy in early ovarian cancer. JAMA 1983; 250: 3072-6.

37. Ashworth A. A synthetic lethal therapeutic approach: poly (ADP) ribose polymerase inhibitors for the treatment of cancers deficient in DNA double-strand break repair. J Clin Oncol 2008; 26: 3785-90.

38. Audeh MW, Penson RT, Friedlander M, Powell B, Bell-McGuinn $\mathrm{KM}$, Scott $\mathrm{C}$, et al. Phase II trial of the oral PARP inhibitor olaparib (AZD2281) in BRCA-deficient advanced ovarian cancer. 2009 ASCO Annual Meeting, Abstract 5500.

39. Chu QS. Aflibercept (AVE0005): an alternative strategy for inhibiting tumour angiogenesis by vascular endothelial growth factors. Expert Opin Biol Ther 2009; 9: 263-71.

40. Vergote I, Amant F, Advani S, Goswani C, Hirte H, Provencher D, et al. Aflibercept (VEGF-TRAP) in advanced ovarian cancer patients with recurrent symptomatic malignant ascites: results of a randomized, double-blind, placebo-controlled study. The 16th ESGO International Meeting 2009, Abstract 1428.

41. Jacobsen PB, Bovbjerg DH, Redd WH. Anticipatory anxiety in women receiving chemotherapy for breast cancer. Health Psychol 1993; 12: 469-75.

42. Morrow GR, Hickok JT, Rosenthal SN. Progress in reducing nausea and emesis: comparisons of ondansetron (Zofran), granisetron (Kytril), and tropisetron (Navoban). Cancer 1995; 76: 343-57.

43. Ryan JL, Heckler C, Dakhil SR, Kirshner J, Flynn PJ, Hickok JT, et al. Ginger for chemotherapy-related nausea in cancer patients: A URCC CCOP randomized, double-blind, placebo-controlled clinical trial of 644 cancer patients. 2009 ASCO Annual Meeting, Abstract 9511.

44. Hickok JT, Roscoe JA, Morrow GR, Ryan JL. A phase II/III randomized, placebo-controlled, double-blind clinical trial of ginger (Zingiber officinale) for nausea caused by chemotherapy for cancer: a currently accruing URCC CCOP Cancer Control Study. Support Cancer Ther 2007; 4: 247-50. 